\title{
Análisis de los procesos vinculados a la gestión de seguridad y salud en el trabajo
}

\author{
Analysis of the processes linked to the management of safety and health \\ at work
}

\section{Análise dos processos relacionados com a gestão da saúde e segurança no trabalho}

\section{Andrea Querales \\ andreiquerales@gmail.com \\ ORCID 0000-0002-4835-2860}

Universidad del Zulia, Núcleo Costa Oriental del Lago, Venezuela

Artículo recibido 4 septiembre 2020 | Arbitrado del 3 al 14 octubre 2020 | Publicado el 1 enero 2021

RESUMEN

En este artículo se presenta un análisis de los procesos vinculados a la gestión de seguridad y salud en el trabajo; esta investigación forma parte de un proyecto más amplio dirigido a evaluar la gestión de seguridad y salud en el trabajo en las empresas que construyen localizaciones para la industria petrolera en el estado Zulia. Se clasificó como descriptiva, con diseño no experimental, transeccional y de campo. Se aplicó un cuestionario con escala de frecuencia, validado por el juicio de expertos y se aplicó el coeficiente estadístico Alfa de Crombach para el cálculo de su confiabilidad, cuyo valor fue 0,95. El análisis de los resultados se realizó mediante la media aritmética. Se concluye que en las organizaciones estudiadas la gestión de seguridad y salud en el trabajo han asumido un conjunto de procesos integrados vinculados a dicha gestión, logrando garantizar el logro de los objetivos organizacionales.

Palabras clave: Gestión de seguridad y salud en el trabajo; industria petrolera; procesos; procesos de protección, procesos preventivos
ABSTRACT

RESUMO
Project management in civil construction This article presents an analysis of the processes related to the management of health and safety at work; this research is part of a larger project aimed at evaluating occupational health and safety management in companies that build locations for the oil industry in Zulia state. It was classified as descriptive, with a non-experimental, transectional and field design. A questionnaire with a frequency scale was applied, validated by the judgment of experts and the statistical coefficient Alpha of Crombach was applied to calculate its reliability, whose value was 0.95 . The analysis of the results was carried out using the arithmetic mean. It is concluded that in the organizations studied, occupational health and safety management have assumed a set of integrated processes linked to said management, managing to guarantee the achievement of organizational objectives.

Key words: Occupational health and safety management; oil industry; processes; protection processes; preventive processes
Este artigo apresenta uma análise dos processos relacionados à gestão da segurança e saúde ocupacional; esta pesquisa faz parte de um projeto mais amplo que visa avaliar a gestão da segurança e saúde ocupacional em empresas que constroem locais para a indústria de petróleo no estado de Zulia. Foi classificado como descritivo, com desenho não experimental, transversal e de campo. Foi aplicado um questionário com uma escala de frequência, validado pelo julgamento de especialistas e aplicado o coeficiente estatístico Alfa de Crombach para o cálculo de sua confiabilidade, cujo valor foi de 0,95. A análise dos resultados foi realizada pela média aritmética. Conclui-se que nas organizações estudadas, a gestão da segurança e saúde ocupacional assumiu um conjunto de processos integrados ligados a essa gestão, conseguindo garantir o cumprimento dos objetivos organizacionais.

Palavras-chave: Gestão da segurança e saúde ocupacional; indústria do petróleo; processos; processos de proteção; processos preventivos 


\section{INTRODUCCIÓN}

A nivel mundial, la seguridad industrial y salud en el trabajo es considerada como la columna vertebral que identifica, analiza, controla los niveles de riesgos y peligrosidad existentes en diversos contextos y medios ambientes laborales. La misma constituye un derecho humano vital que forma parte del programa para el desarrollo organizacional centrado en personas y figuran como la base de toda empresa sostenible que pretenda lograr mayor productividad, calidad y motivación en sus empleados.

Partiendo de la necesidad básica del resguardo de la integridad de la sociedad en su desarrollo laboral, los países suscriben convenios internacionales, establecen leyes y decretos a fin de propiciar que todas las organizaciones (empresas públicas o privadas, cooperativas $\mathrm{u}$ otras formas asociativas de carácter productivo o de servicio) cumplan con las normativas técnicas y legales necesarias para garantizar el bienestar integral de los trabajadores, abarcando las dimensiones físicas, psicológicas y sociales, así como la generación de un medio ambiente de trabajo seguro.

En Latinoamérica, la seguridad y salud en el trabajo ha recibido poca atención debido a la carencia de conciencia generalizada, de raíces culturales, en cuanto a la importancia de un ámbito de trabajo sano, seguro, a la debilidad y/o incapacidad de las instituciones responsables de promover así como de hacer cumplir mejores condiciones de trabajo, verificándose fallas considerables en la aplicación de los sistemas de gestión en materia de seguridad y salud en el trabajo

En Venezuela, según establece la Norma Técnica NT-01-2008, el estado crítico de la seguridad y salud en el trabajo viene dada por una serie de factores políticos, económicos, sociales y culturales que han derivado en daños o deterioro a la salud de los trabajadores, entre otras cosas, por las diferentes formas, condiciones y esquemas de organización del trabajo no controlado.

De allí, el esfuerzo de distintas Instituciones Venezolanas en aumentar el nivel de concientización en materia de cultura preventiva, como es el Instituto Nacional de Prevención, Salud y Seguridad Laborales (INPSASEL), además de gestionar, promover y verificar el cumplimiento en el ámbito nacional de los lineamientos establecidos tanto en la Constitución como en las leyes venezolanas vinculadas: Ley Orgánica de Prevención, Condiciones y Medio Ambiente de Trabajo (LOPCYMAT, 2005), Reglamento Parcial LOPCYMAT (2007), conjuntamente con normativas internacionales en prevención de riesgos y decretos aprobados en el área de estudio, que producen un efecto estandarizador de derechos, como es el caso del derecho a la seguridad industrial y salud ocupacional en el trabajo.

Ante la situación precedente, es relevante que la alta gerencia dote a la empresa de la estructura organizativa apropiada, proporcione el marco de políticas que guíe la acción y fomenten una cultura organizativa que inculque, de palabra y obra, la integridad entre todos los empleados a la hora de realizar sus actividades diarias. Es importante contar con una estructura organizativa independiente de líneas de negocios $u$ operacionales, especializada y dedicada a la gestión de seguridad y salud en el trabajo.

En este contexto, se consideró relevante realizar un análisis de los 
procesos vinculados a la gestión de seguridad y salud en el trabajo en las empresas que construyen localizaciones para la industria petrolera en el estado Zulia, que según Henao (2016), pueden clasificarse en dos (2) grupos: preventivos y de protección. En los primeros, suelen englobarse: prevención de accidentes, ergonomía laboral, medicina ocupacional, y la administración de seguridad industrial.

Mientras, los de protección: contra incendios, de bienes y personalidades, y ambiental. Agrega este autor, son procesos que se mantienen interrelacionados estrechamente siendo, a su vez, interdependientes. Su interacción obliga a que cualquier cambio producido en uno de ellos, influya en los otros, lo cual originará nuevos cambios en los demás y generará adaptaciones y ajustes en todo el sistema. Así entonces, el estudio profundizó en conceptos relacionados con la gestión de la seguridad y salud en el trabajo, permitiendo constatar la teoría del análisis con la práctica.

\section{MÉTODO}

El presente estudio se categorizó como de tipo descriptivo. Al respecto, Sabino (2014) afirma, el tipo descriptivo se centra en recoger información relacionada con el estado real de las personas, objetos, situaciones o fenómenos, y con base a esta información describir el estado actual del fenómeno estudiado. De igual manera, se enmarcó en la metodología cuantitativa, bajo un diseño de tipo no experimental, de campo y transeccional.

En relación a la población objeto de estudio, fueron seleccionadas tres (3) empresas que construyen localizaciones para la industria petrolera en el estado Zulia, Venezuela activas al momento de la ejecución de este trabajo. Los datos se recolectaron a través de los gerentes, coordinadores, supervisores, líderes de proyectos e inspectores de las gerencias de seguridad y salud en el trabajo y las gerencias de operaciones en las empresas referidas, estimándose un total de 72 sujetos informantes.

Se aplicó un cuestionario estructurado con respuestas estandarizadas configuradas bajo una escala de medición de frecuencias validado por el juicio de expertos y se aplicó el coeficiente estadístico Alfa de Cronbach para el cálculo de su confiabilidad, cuyo valor fue 0,95 cuyo resultado indicó alta confiabilidad, según la escala de valoración utilizada, indicando que el instrumento era apto para ser aplicado. El análisis de los resultados se realizó mediante la media aritmética.

\section{RESULTADOS}

Para el estudio de los procesos vinculados a la gestión de seguridad y salud en el trabajo, ésta fue dividida en dos (2) dimensiones, según lo establecido por los autores consultados: procesos preventivos y de protección; los cuales se desglosan seguidamente.

A tal efecto, en la tabla 1 se resumen los resultados para la dimensión, con una media de 2,69, implicando alta presencia de los procesos vinculados a la gestión de seguridad y salud en el trabajo que realizan en las empresas que construyen localizaciones para la industria petrolera en estudio, delineándola como fortalezas. De manera específica, se evidencia alta presencia tanto de los procesos preventivos (2,67), como de los procesos de protección $(2,71)$ constituyéndolos en fortalezas de su gestión de seguridad y salud en el trabajo. 
Estos resultados, tienen congruencia con lo enunciado al respecto por Gómez (2017), quien refiere que en las organizaciones la gestión de seguridad y salud en el trabajo debe corresponder a un conjunto de procesos integrados que garanticen el logro de objetivos organizacionales, apareados al logro de la integridad física y emocional de sus trabajadores en las actividades operativas. Señala este autor, esta gestión se organiza en una serie de procesos destinados a la prevención de riesgos (seguridad) y la protección organizativa (salud en el trabajo).

Tabla 1. Procesos vinculados a la gestión de seguridad y salud en el trabajo

\begin{tabular}{ccc}
\hline INDICADORES & MEDIA & CATEGORÍA \\
\hline Procesos preventivos & 2,67 & Alta presencia/fortaleza \\
Procesos de protección & 2,71 & Alta presencia/fortaleza \\
DIMENSIÓN & $\mathbf{2 , 6 9}$ & Alta presencia/fortaleza \\
\hline
\end{tabular}

De tal forma, para la investigadora, en las empresas que construyen localizaciones para la industria petrolera en el estado Zulia, se está garantizando la efectiva realización de los procesos involucrados a la gestión de seguridad y salud en el trabajo, al calificarse con alta presencia en su gestión; lo cual hace inferir que están viendo dichos procesos como el conjunto de acciones pertinentes para su eficiente cumplimiento, contribuyendo a la prevención de riesgos y a la protección industrial, siendo estas acciones los procesos preventivos y procesos de protección.

En lo concerniente al detalle, de la dimensión procesos preventivos utilizada para medir los procesos vinculados a la gestión de seguridad y salud en el trabajo, de seguido se especifican los resultados obtenidos al respecto, mostrados en la tabla 2 , la cual recoge el resultado de la dimensión $(2,67)$ y cada uno de sus indicadores.
De esta forma, se evidencia alta presencia para todos los ítems aplicados en cada uno de los indicadores: prevención de accidentes $(2,69)$, ergonomía laboral $(2,61)$, medicina ocupacional (2,51), y la administración de seguridad industrial $(2,86)$, siendo estas actividades medidas por los ítems aplicados fortalezas para su gestión de seguridad y salud en el trabajo, en lo que refiere a los procesos preventivos como parte de los procesos vinculados a dicha gestión.

Este resultado de alta presencia de los procesos preventivos, valida a Alonso (2016) cuando asevera, el objetivo central de la política de prevención en la empresa debe ser el desarrollo de una cultura preventiva, en la que se busquen unas condiciones de trabajo adecuadas, donde las personas -principal valor de la empresase conviertan también en objetivo empresarial. 
Tabla 2. Procesos preventivos

\begin{tabular}{cc}
\hline & Indicador: Prevención de accidentes \\
\hline $\mathbf{N}^{\circ}$ & ÍTEMS
\end{tabular}

1. Los trabajadores comprenden su papel en el proceso de prevención de accidentes, asumiendo su responsabilidad sobre los esfuerzos que realiza la organización para protegerlos.

2. Contemplan las actividades preventivas como una actividad a gestionar.

3. El proceso de prevención de accidentes que realizan busca como objetivo principal la reducción de los índices de accidentabilidad.

\section{Promedio Indicador}

\section{Indicador: Ergonomía laboral ÍTEMS}

4. Adaptan el trabajo a las capacidades del ser humano, como parte de la política de prevención de accidentes.

5. Se controla la introducción de las nuevas tecnologías a la adaptación de las capacidades de la población laboral existente.

6. Establecen condiciones ergonómicas en la adquisición de útiles de trabajo

\section{Promedio Indicador}

\section{Indicador: Medicina ocupacional} ÍTEMS

7. Aplican programas de entrenamiento, a través del departamento de medicina ocupacional, para la divulgación de las normas que permiten la prevención de accidentes.

8. Se desarrollan programas de medicina ocupacional compuestos de una serie de planes que giran en torno a la salud de los empleados.

9. Se proveen de información periódica sobre las exposiciones de los trabajadores, a través de mediciones higiénicas.

\section{Promedio Indicador}

\section{Indicador: Administración de la seguridad} ÍTEMS

10. Se establecen actividades grupales que conllevan a mejorar los diferentes procesos de seguridad.

11. Se divulgan en toda la organización las políticas de seguridad industrial.

12. Actualizan periódicamente sus programas de seguridad y salud en el trabajo

Promedio Indicador

Promedio dimensión
MEDIA CATEGORÍA

2,51 Alta presencia

Fortaleza

2,72 Alta presencia

Fortaleza

2,85 Alta presencia

Fortaleza

2,69 Alta presencia Fortaleza

MEDIA CATEGORÍA

2,74 Alta presencia

Fortaleza

2,51 Alta presencia

Fortaleza

2,58 Alta presencia Fortaleza

2,61 Alta presencia Fortaleza

MEDIA CATEGORÍA

2,61 Alta presencia

Fortaleza

2,57 Alta presencia

Fortaleza

2,35 Alta presencia Fortaleza

2,51 Alta presencia Fortaleza

MEDIA CATEGORÍA

2,71 Alta presencia Fortaleza

2,96 Alta presencia Fortaleza

2,92 Alta presencia

Fortaleza

2,86 Alta presencia Fortaleza

2,67 Alta presencia Fortaleza 
En cuanto a los procesos preventivos, la investigadora infiere, en las empresas que construyen localizaciones para la industria petrolera en el estado Zulia, dan seguimiento al cumplimiento efectivo de estos procesos preventivos, por lo cual están asumiendo que la prevención no es parte del trabajo que se pueda delegar en otro, sino un componente del trabajo que no se puede separar del mismo.

En el caso de la otra dimensión, considerada para medir los procesos vinculados a la gestión de seguridad y salud en el trabajo, denominada procesos de protección, se muestran los hallazgos encontrados en la tabla 3 , donde se especifican los indicadores aplicados para su medición. Al respecto, se observa una media para la dimensión de 2,71, signando los procesos de protección con alta presencia en su gestión de seguridad y salud en el trabajo, implicando fortalezas.

Tabla 3. Procesos de protección

\section{Indicador: Protección contra incendios}

\begin{tabular}{cc}
\hline $\mathbf{N}^{\circ}$ & ÍTEMS \\
13. La gerencia actualiza periódicamente el conjunto de medidas que
\end{tabular} se disponen para proteger la organización contra la acción del fuego

14. Preparan a los trabajadores en técnicas de prevención que les permitan el combate de los conatos e incendios declarados que se originen.

15. Se inspeccionan los procedimientos operativos que por su naturaleza originan incendios para tomar medidas de prevención efectivas.

\section{Promedio Indicador}

\section{Indicador: Protección de bienes y personalidades}

$\mathbf{N}^{\circ}$ ÍTEMS

16. Diseñan sistemas protectores para garantizar la seguridad de la empresa en su amplio contexto.

17. Aplican normas de actuación a los inspectores de seguridad integral, para el respeto de los derechos humanos de trabajadores.

18. Se realiza un programa de recorridos (rondas diurnas y nocturnas) donde los encargados visualicen las instalaciones asegurándose que todo se encuentre normal.

\section{Promedio Indicador}

\section{MEDIA CATEGORÍA}

Alta presencia

2,74 Fortaleza

2,78

Alta presencia

Fortaleza

Alta presencia

Fortaleza

\section{2,75 Alta presencia}

Fortaleza

MEDIA CATEGORÍA

Alta presencia

2,72 Fortaleza

2,68 Alta presencia Fortaleza

2,74 Alta presencia Fortaleza

2,75 Alta presencia Fortaleza 


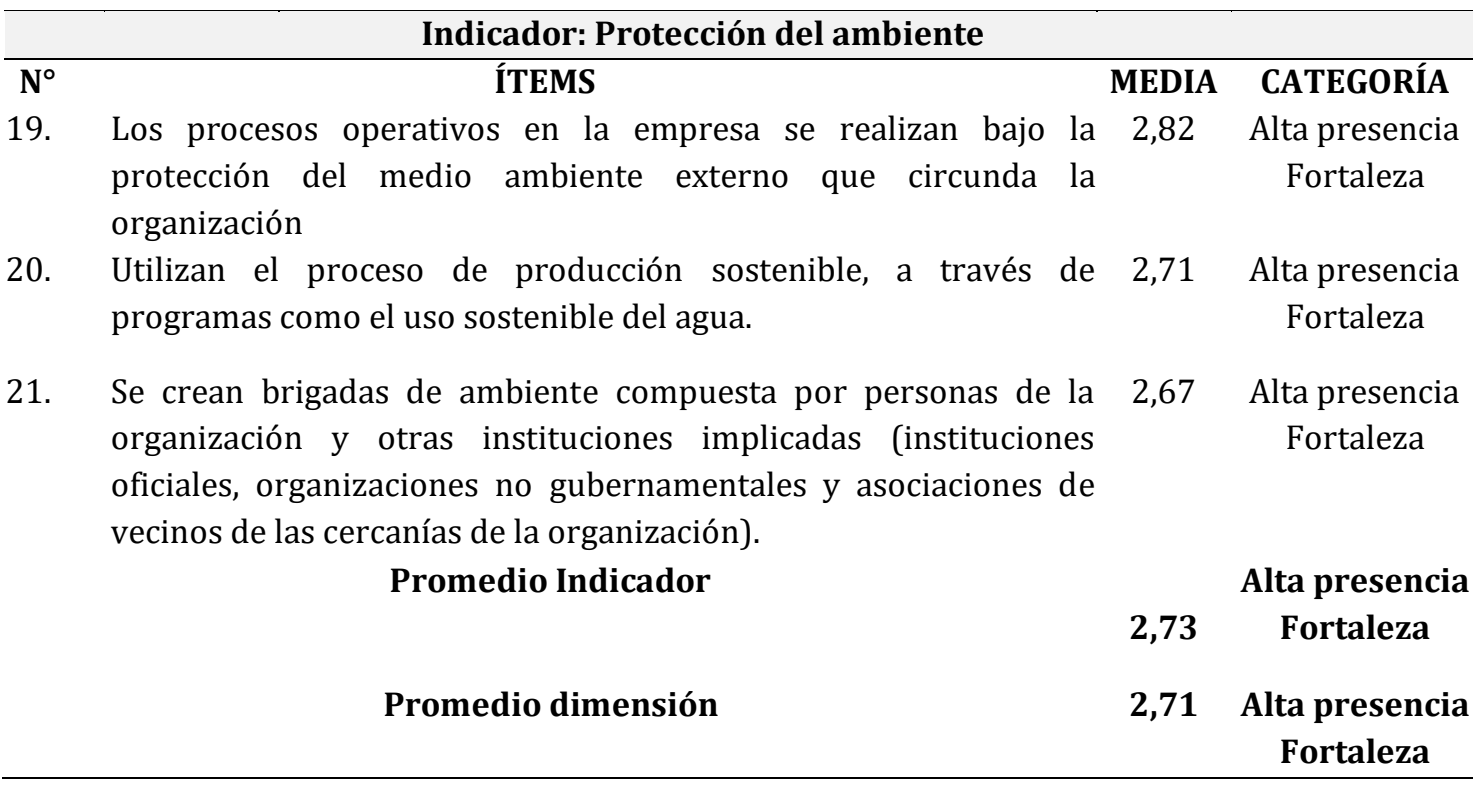

Esta situación es explicada por la alta presencia otorgada a cada uno de los procesos de protección medidos, con medias que así lo ratifican: protección contra incendios $(2,75)$, protección de bienes y personalidades (2,75), y protección del ambiente $(2,73)$.

Así entonces, su categoría de alta presencia valida a Diez y González (2014) cuando explican, los procesos de protección en empresas deben estar dirigidos a salvaguardar la vida y salud de todos los integrantes de la misma, así como la preservación de las instalaciones, bienes y ambiente. De allí, la gerencia de seguridad y salud en el trabajo debe realizar procesos de protección dirigidos a la protección contra incendios, protección de bienes y personalidades y protección del ambiente.

Con lo evidenciado la investigadora puede inferir, en las empresas que construyen localizaciones para la industria petrolera en el estado Zulia, la gerencia de seguridad y salud en el trabajo asume entre sus responsabilidades planificar, organizar, ejecutar y controlar los procesos de protección, a fin de proteger al recurso más importante para las mismas (los trabajadores); dando cumplimiento a las normas de protección contra los riesgos profesionales en las operaciones.

\section{CONCLUSIONES}

En las organizaciones estudiadas la gestión de seguridad y salud en el trabajo ha asumido un conjunto de procesos integrados vinculados a dicha gestión, logrando garantizar el logro de los objetivos organizacionales, apareados al logro de la integridad física y emocional de sus trabajadores en las actividades operativas. Se evidencia como esta gestión se organiza en una serie de procesos destinados a la prevención de riesgos (seguridad) y la protección organizativa (salud en el trabajo). 
REFERENCIAS

Alonso, G. (2016). Documentación del sistema de gestión en seguridad y salud en el trabajo. Universidad de Pereira. Postgrado: Facultad de Ingeniería Industrial. Programa de Maestría en Gestión de la Calidad y Normalización Técnica. Colombia.

Diez, A. y González, A. (2014). Seguridad y la protección medioambiental en la gestión y supervisión del montaje $\mathrm{y}$ mantenimiento de sistemas de automatización industrial. Editorial Paraninfo. España.

Gómez, B. (2017). Manual de prevención de riesgos laborales. Ediciones Marge Books.Barcelona. España.
Henao, F. (2016). Seguridad y salud en el trabajo: Conceptos básicos. Tercera edición. Ediciones ECOE. Colombia.

LOPCYMAT (2005). Artículo 82. Reglamento parcial de la Ley orgánica prevención condiciones y medio ambiente de trabajo.

LOPCYMAT. (2007). Reglamento parcial de la Ley orgánica prevención condiciones y medio ambiente de trabajo. En: http://www.inpsasel.gob.ve/moo_news /lopcymat.html

Norma Técnica Programa de Seguridad y Salud en el Trabajo (NT-01-2008). INPSASEL: Disponible en: http://www.medicinalaboraldevenezuel a.com.ve/archivo 